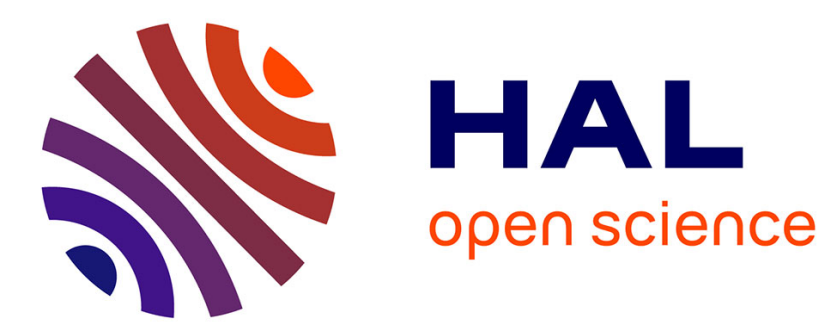

\title{
Entre ordonnancement hiérarchisé des professions et regroupement des métiers par domaine d'activité. La double asymétrie des représentations ordinaires
}

Thomas Amossé, Pénissat Etienne

\section{- To cite this version:}

Thomas Amossé, Pénissat Etienne. Entre ordonnancement hiérarchisé des professions et regroupement des métiers par domaine d'activité. La double asymétrie des représentations ordinaires. Année Sociologique, 2019, 69 (2), pp.511-539. 10.3917/anso.192.0511 . hal-03323712

\author{
HAL Id: hal-03323712 \\ https://hal.science/hal-03323712
}

Submitted on 22 Aug 2021

HAL is a multi-disciplinary open access archive for the deposit and dissemination of scientific research documents, whether they are published or not. The documents may come from teaching and research institutions in France or abroad, or from public or private research centers.
L'archive ouverte pluridisciplinaire HAL, est destinée au dépôt et à la diffusion de documents scientifiques de niveau recherche, publiés ou non, émanant des établissements d'enseignement et de recherche français ou étrangers, des laboratoires publics ou privés. 


\title{
Entre ordonnancement hiérarchisé des professions et regroupement des métiers par domaine d'activité. La double asymétrie des représentations ordinaires
}

\author{
Thomas Amossé (Cnam, Lise, CEET), Etienne Pénissat (CNRS, Ceraps)
}

\begin{abstract}
Résumé :
A partir d'un dispositif d'enquête sur tablette numérique, cet article étudie les représentations ordinaires de l'espace social. Comment les individus délimitent-ils deux milieux sociaux dans un ensemble fermé de 11 professions appartenant à une fraction limitée de l'espace social ? Réalisés successivement sur des professions classiquement considérées comme relevant du « haut » et du «bas » de l'espace social, les classements des enquêtés démontrent en premier lieu la force toujours actuelle de l'opposition entre les professions d'employés et d'ouvriers : comme points saillants, elles délimitent le principal clivage interne aux classes populaires. Pour les professions de cadres et de chefs d'entreprise ensuite, trois représentations (positionnelle, statutaire et fonctionnelle) se dégagent des classements réalisés, dont la première est clairement hiérarchisée. L'opposition selon le secteur public et privé se retrouve dans les deux séquences de façon transversale. L'article conclut à une double asymétrie des représentations ordinaires : les hiérarchies professionnelles et sociales sont plus souvent évoquées pour et par les individus occupant une position élevée dans la société, alors que ce sont davantage des oppositions transversales (statutaires ou fonctionnelles) qui sont utilisées pour et par ceux situés plus bas dans l'échelle des emplois et des positions sociales.
\end{abstract}

Mots clés : Métiers - Professions - Représentations ordinaires - Frontières sociales - Hiérarchies sociales et professionnelles - Statut d'emploi

\section{Résumé anglais :}

\section{Hierarchical ordering or grouping by field of activity? The double asymmetry of ordinary representations on occupations}

Based on a digital tablet survey system, this article investigates the ordinary representations of social space. How do individuals delimit two social groups among a closed set of 11 occupations to a limited fraction of the social space? The respondents' classifications, carried out successively on occupations classically considered as belonging to the "top" and "bottom" of the social space, demonstrate first of all the ever-present strength of the opposition between the occupations of "employés" (clerical or public) and of "ouvriers" (manual): they function as salient points that delimit the main internal cleavage between the working classes. For the executives and business managers occupations, three representations (positional, statutory and functional) emerge of the classifications, the first of which is clearly hierarchical. The opposition between the public and private sector plays a transversal role in both games. The article concludes that there is a double asymmetry in ordinary representations: professional and social hierarchies are more often mentioned for and by individuals occupying a higher position in society, while transversal oppositions (statutory or functional) are more often used for and by those who are at a lower level in the scale of occupations and social positions.

Keywords: Occupations - Ordinary Representations - Social Boundaries - Social and Professional Hierarchies - Employment Status 
Au début des années 1980, dans le cadre de la refonte de la nomenclature des Catégories SocioProfessionnelles (CSP $\left.{ }^{1}\right)$, Luc Boltanski et Laurent Thévenot (2015 [1983]) conçoivent un ensemble de dispositifs ludiques et expérimentaux pour enquêter sur les manières dont est perçue la société française. Leurs résultats mettent en lumière une convergence des représentations ordinaires avec les catégories statistiques, juridiques et politiques de division du monde social. Ils soulignent toutefois que l'espace social tels que se le représentent les enquêtés ne s'organise pas de façon homogène, segmentée et orientée. Il s'apparente à une classification « naturelle », non à une classification « logique » telle celle du botaniste Carl von Linné qui, au XVIIIème siècle, a élaboré un système classificatoire des végétaux en croisant strictement les critères utilisés pour leur identification ${ }^{2}$. Les classifications naturelles tiennent quant à elles davantage du bricolage : elles reprennent les catégories sociales, les images issues de l'expérience sensible, de la vie quotidienne. Mises en évidence dans les jeux de L. Boltanski et L. Thévenot, les représentations ordinaires s'organisent ainsi autour de «points saillants » qui, durcis par un travail politique de représentation des univers professionnels et sociaux, servent d'appuis cognitifs, de passerelles vers les catégories instituées : l'« ouvrière d'usine emballeuse » fait largement consensus entre enquêtés pour désigner les «travailleurs non qualifiés »; le «fraiseur P2 » est le représentant paradigmatique des «ouvriers qualifiés », de même que l'« ingénieur travaux publics » l'est pour les «cadres» (id., p. 11). A côté de ces points saillants, de larges «zones floues » regroupent des professions perçues comme à la frontière des catégories instituées, là où certains critères perdent de leur pertinence, parfois au profit d'autres, ou entrent en contradiction.

C'est précisément pour mieux comprendre la topographie des représentations ordinaires de l'espace social, entre points saillants et zones frontières, que nous avons élaboré un dispositif d'enquête original ${ }^{3}$. S'inspirant des jeux originels de L. Boltanski et L. Thévenot, et se situant dans le prolongement de premières adaptations en France comme à l'étranger (Pénissat et al., $2015 ; c f$. Pénissat et al., 2016 pour un premier recensement), il s'est appuyé sur l'infrastructure française d'enquête quantitative sur tablette numérique pour les sciences sociales (Elipss) ${ }^{4}$. Le questionnaire demandait aux participants de classer 11 professions $^{5}$ (cf. encadré 1) en suivant les consignes suivantes : «le jeu consiste à créer deux groupes différents en mettant ensemble les métiers qui vous semblent correspondre au même milieu social. Si des métiers vous semblent trop différents des autres, vous pouvez les laisser dans la zone de départ, à gauche [de l'écran de la tablette]. Il n'y a pas de bonne ou mauvaise réponse : faites comme bon vous semble ». Une fois les deux groupes constitués, l'enquêté devait choisir «le métier qui représente le mieux » chacun d'eux puis leur « donner un nom ». Enfin, deux questions lui étaient posées, qui visaient à comprendre comment il avait procédé pour classer les métiers : Il lui était demandé si, «pour constituer ses groupes, il avait pensé à des personnes qu'il connaissait/à son propre cas / à des personnes vues à la télévision, dans la presse, etc. / avait réfléchi à partir de critères généraux (revenu, diplôme, situations de travail, etc.) / écouté son intuition ou procédé au hasard » (plusieurs réponses étant autorisées) ; la dernière question, également à choix multiples, permettait aux enquêtés d'indiquer les informations sur lesquelles ils s'étaient appuyés pour réaliser les groupes, une liste de treize critères

\footnotetext{
${ }^{1}$ La nomenclature socio-professionnelle est désignée par le sigle CSP de 1954 à 1982 et, depuis, par celui de PCS (pour Profession et Catégories Socio-professionnelles).

${ }^{2}$ L'opposition de ces deux formes de classification a été initialement analysée par Alain Desrosières et Laurent Thévenot dans les travaux de refonte de la nomenclature socio-professionnelle (1979, p. 49 à 52).

${ }^{3}$ Ce projet a initialement été coordonné par Cécile Brousse et a associé, outre les auteurs de l'article, Jérôme Deauvieau, Ivailo Petev, Rémi Sinthon et Laurent Thévenot.

${ }^{4}$ L'enquête, réalisée en juillet et septembre 2014, s'inscrit dans le projet «Catégorisations et connaissances ordinaires de la société », qui a bénéficié de l'appel à enquête d'Elipss (http:/quanti.dime-shs.sciencespo.fr/fr/actu/, consulté le 28 novembre 2018).

${ }^{5}$ Les 11 métiers à classer dans le jeu étaient, en séquence 1 : préfet, consultant indépendant, patron (fabrique de conserves), professeur de français (lycée), responsable transport (mairie), médecin généraliste, gérant (magasin de bricolage), ingénieur-informaticien, directrice des ressources humaines, analyste financier (grande banque) et inspectrice des impôts; et en séquence 2 : assistante maternelle, secrétaire comptable (mairie), ouvrière de découpe de viandes, employée de banque, agent de service hospitalier, réparateur d'ascenseur, caissière, chauffeur-livreur, policier municipal, artisan-maçon, soudeur.
} 
étant proposée ${ }^{6}$ L'opération était reproduite deux fois ${ }^{7}$ : sur des métiers d'employés et d'ouvriers (et, dans un cas, d'artisan) ; et sur des métiers de cadres et de chefs d'entreprise (et dans un cas de profession intermédiaire $)^{8}$. Classiquement considérées en sociologie comme relevant respectivement des fractions «basse » et « haute » de l'espace social, ces deux listes de métiers n'étaient pas présentées comme telles aux enquêtés. Elles leur étaient simplement soumises, sans indication particulière ni consigne spécifique.

Les caractéristiques du dispositif, et notamment le choix d'y explorer deux fractions distinctes, et contrastées, de l'espace social ne doivent rien au hasard. Elles résultent en premier lieu de contraintes empiriques, mais offrent dans le même temps l'opportunité de tester de nouvelles hypothèses par rapport aux enquêtes antérieures. De fait, la possibilité ouverte par Elipss de faire passer une enquête expérimentale s'appuyant sur des manipulations tactiles (le déplacement des vignettes de métiers, ou professions ${ }^{9}$, correspondant à celui de fiches cartonnées dans le jeu originel) à un échantillon représentatif de la population française $(\mathrm{n}=782)^{10}$ s'est accompagnée de la nécessité d'adapter le design du jeu. Alors que l'enquête de L. Boltanski et L. Thévenot, ou celles s'en étant inspirées, s'appuyaient sur des cartes comportant de nombreuses informations individuelles (prénom, profession, âge, statut d'emploi, diplôme, etc.), le dispositif mobilisé dans l'article s'est limité à des intitulés de métier pour permettre la passation sur tablette numérique. Pour les mêmes raisons, les nombres de vignettes et de groupes possibles ont été également réduits (respectivement à 11 et $2^{11}$ ), alors les enquêtes antérieures invitaient les enquêtés à classer plusieurs dizaines de cartes, correspondant à l'intégralité de l'espace social, en au plus 10 groupes. Cette restriction des informations manipulées et du spectre de l'analyse a conduit à un déplacement des objectifs poursuivis. Avec un tel dispositif, il n'est plus question d'investiguer, de façon ouverte, les représentations d'ensemble de l'espace social, mais d'examiner les segmentations internes à une ou plusieurs de ses fractions. La logique binaire invite à reformuler la question de recherche en terme de frontière : comment les enquêtés délimitent-ils deux milieux sociaux dans un ensemble fermé, et réduit, de professions appartenant à une zone précise de l'espace social ? Où passe la frontière considérée comme la plus pertinente, et à quel(s) critère(s) renvoie-t-elle ?

\footnotetext{
${ }^{6}$ «Le fait d'être salarié, à son compte ou patron ; le domaine d'activité ; la qualification; le type de contrat (CDI, $C D D$, intérim, au noir...) ; le secteur (public ou privé) ; le revenu ; le style de vie (vêtements, loisirs, intérêts...); le prestige du métier ; le fait d'être chef ou exécutant ; les responsabilités ; le fait qu'il s'agisse d'un métier plutôt masculin ou plutôt féminin; le nombre de salariés (grande, petite entreprise, etc.) ; la pénibilité ou la répétitivité du travail».

${ }^{7}$ Les deux séquences du jeu suivaient un ordre aléatoire pour éviter que la manière de classer le premier ensemble de professions n'influence de façon systématique la manière de classer le second, et réciproquement. Pour neutraliser les effets de liste, l'ordre d'apparition des onze vignettes de métier (de haut en bas) dans chacune des deux séquence était également aléatoire.

${ }^{8}$ Dans la suite de l'article, par simplicité, nous les désignerons respectivement par « employés et ouvriers » et « cadres et chefs d'entreprise ».

${ }^{9}$ Dans la suite de l'article, pour faciliter la lecture, on utilise indistinctement les termes de « profession » et de «métier » dans l'article, bien que les consignes d'enquête aient uniquement utilisé celui de « métier ».

${ }^{10}$ Par rapport aux données mises à disposition par Elipss $(\mathrm{n}=809)$, l'échantillon analysé exclut les 27 observations pour lesquelles les données relatives aux classements et aux caractéristiques individuelles sont manquantes.

${ }^{11}$ Pour assouplir cette règle, il était tout de même possible pour les enquêtés de laisser à part certaines des professions sans les classer, ce que 112 (respectivement 175) enquêtés ont fait dans le jeu portant sur les ouvriers et employés (respectivement les cadres et chefs d'entreprise).
} 


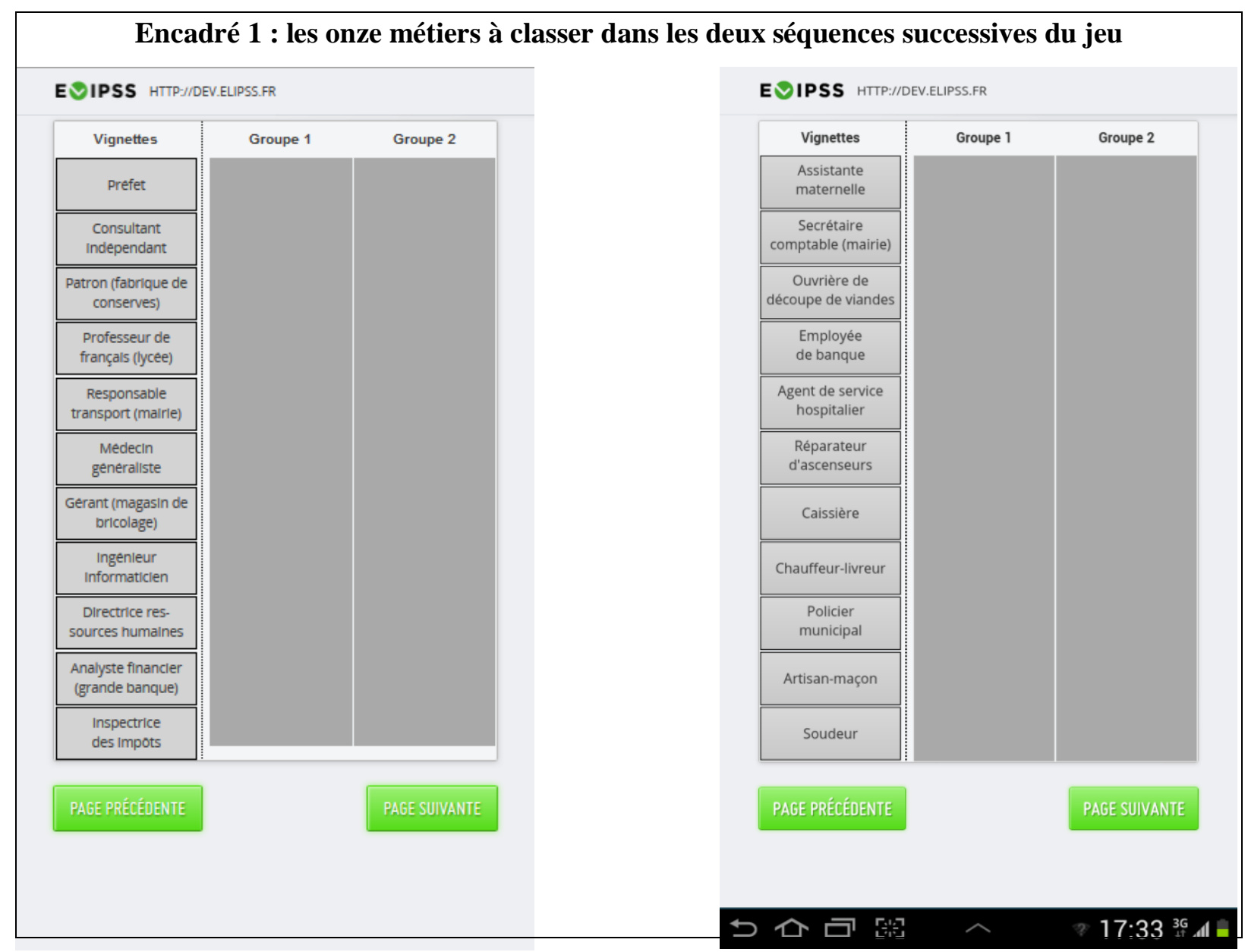

Cette reformulation de la problématique du jeu répond à certains défis posés par l'évolution de la société et du regard porté sur elle. Au cours des dernières décennies, et plus particulièrement dans les années 1980 et 1990, un nouveau modèle d'organisation des métiers et professions s'est en effet développé dans les conventions collectives : avec les grilles à critères classants (que sont par exemple le niveau de responsabilité, le degré d'autonomie, le type d'activité et les connaissances requises) ${ }^{12}$, les emplois sont ordonnés sur une échelle unique allant des plus basses qualifications jusqu'aux plus élevées. Ces grilles de conventions collectives, alors adoptées dans un nombre croissant de branches et d'entreprises, se sont accompagnées du succès des logiques « compétence » (Tallard, 2001 ; Coutrot, 2005) et du vocabulaire de la responsabilité (Burnod et Chenu, 2003). Les catégories historiques des conventions collectives, dites Parodi, ont ainsi perdu de leur force symbolique avec cette diffusion de grilles de classification des professions inspirées d'un nouvel esprit, managérial, du capitalisme (Boltanski et Chiapello, 1999). D'une organisation catégorielle selon une logique de métiers, qui étaient simplement listés au sein des groupes « Ouvrier », «Employé technicien agent de maitrise (Etam) » ou « Cadre », on tendrait à passer à une organisation hiérarchisée des emplois occupés, censée permettre une meilleure anticipation des progressions de carrière. L'évolution normative ne s'est pas limitée au droit conventionnel : elle a également affecté, de façon partiellement liée, les instruments statistiques et en premier lieu la nomenclature socio-professionnelle (Amossé, 2013). La pertinence des choix ayant présidé à sa création dans les années 1950 puis à sa refonte en 1982 - de l'intuition des «milieux sociaux » à l'adéquation avec les compromis sociaux de classement (Desrosières, Thévenot, 2002 [1988]) - a ainsi été remise en question par les travaux engagés autour des échelles de prestige d'inspiration nord-américaine (Chambaz, Maurin, Torelli, 1998) ou, au niveau européen, par le succès des schémas de classe stratifiés

\footnotetext{
${ }^{12}$ Pour reprendre les critères retenus dans la première grille de ce type, qui a été conclue en 1975 dans la branche de la métallurgie. La diffusion de ce modèle a connu un mouvement d'accélération dans les deux décennies suivantes.
} 
renvoyant au concept britannique de « relation d'emploi » (Goldthorpe, 2000 ; Brousse, 2012 pour une discussion).

Si elles concernent le droit et la statistique, ces évolutions sont susceptibles d'avoir influencé le regard porté, de façon commune, sur l'organisation des espaces sociaux et professionnels : adopte-t-on, en particulier, une représentation hiérarchisée des professions (en écho aux grilles à critères classants) quand on doit délimiter deux milieux sociaux ? Ou bien préfère-t-on les organiser selon le type de métier exercé (comme dans les catégories Parodi) ? Ou encore selon le statut d'emploi, des travaux récents ayant rappelé l'importance de ce clivage dans les représentations ordinaires en France (Hugrée, de Verdalle, 2015) comme dans les situations concrètes en Europe (Hugrée, Pénissat, Spire, 2015) ? Ces questions se déclinent plus spécifiquement dans les deux fractions d'espace que nous avons investiguées. Dans un contexte de déclin du monde ouvrier industriel (Vigna, 2012) et de développement simultané de l'archipel des employés (Chenu, 1990), de nombreux travaux se sont intéressés à la manière d'organiser la diversité des emplois et positions sociales relevant des classes populaires. On peut ainsi s'interroger sur la force du clivage entre employés et ouvriers, susceptibles d'avoir été remplacé par la segmentation entre travailleurs qualifiés et non qualifiés (Amossé, Chardon, 2006). A l'opposé du spectre social, au-delà de la question controversée de la banalisation des cadres (Bouffartigue, 2001 ; Amossé, 2011), se pose celles de leur segmentation hiérarchique, dont rendrait par exemple compte les différents régimes de réduction du temps de travail, et de leur frontière, supposée de plus en plus poreuse, avec le monde des indépendants. Ces questions seront successivement examinées dans les deux parties composant l'article. On s'y interrogera par ailleurs, de façon transversale, sur la diffusion sociale des représentations hiérarchisées des deux ensembles de métiers considérés. Sans trop anticiper sur nos résultats, nous verrons que les travailleurs eux-mêmes situés en haut de l'échelle des emplois adoptent une représentation plus systématiquement hiérarchique et critérielle que ceux occupant des emplois d'exécution, qui restent davantage attachés à l'intuition de milieux sociaux adossés aux logiques de métiers et environnements de travail.

\section{La force maintenue du clivage employé / ouvrier}

Comment sont classées, désignées et représentées les diverses professions d'ouvriers et d'employés ? Peut-on repérer une pluralité de classements ${ }^{13}$ les concernant, ou au contraire un consensus se dégaget-il quant à la manière de scinder en deux le pan de l'espace socio-professionnel qu'elles occupent ?

Les près de huit cent enquêtés pouvaient potentiellement proposer 1024 classements différents de deux groupes ${ }^{14}$. Bien que le nombre de classements effectivement réalisés dans ce premier jeu soit sensiblement plus faible - on en dénombre 226, dont 146 classements uniques et 80 classements retenus par au moins deux enquêtés et douze par plus de dix enquêtés -, l'analyse ne peut pas s'appuyer sur la comparaison des joueurs ayant strictement réalisé le même classement. Il faut pouvoir décomposer les classements pour en comprendre la logique et construire une mesure de la distance qui les sépare afin de les regrouper et ainsi analyser comment s'organise leur diversité. Dans une première étape, on a adopté une méthode éprouvée dans des travaux antérieurs (Deauvieau et al., 2014), qui consiste à identifier les paires de professions ayant été les plus (respectivement le moins) associées dans un même groupe.

13 On utilisera le terme «classement» pour désigner l'ordonnancement des professions en groupes, indépendamment de leur caractère hiérarchisé ou pas. Il convient de rappeler que ce terme n'est pas utilisé dans l'enquête ( $c f$. supra les consignes aux joueurs).

${ }^{14} \mathrm{Ce}$ nombre, estimé sous l'hypothèse qu'aucun métier n'est laissé à part, correspond de façon équivalente à $2^{\wedge} 11 / 2$ ou à la somme de $\mathrm{C}(2,11), \mathrm{C}(3,11), \mathrm{C}(4,11)$ et $\mathrm{C}(5,11)$, où $\mathrm{C}(\mathrm{i}, \mathrm{j})$ est égal au nombre de combinaisons de i métiers dans un ensemble de $\mathrm{j}$ métiers. Le nombre est très nettement supérieur si on tient compte du fait que les joueurs pouvaient laisser des métiers à part (donc, d'un point de vue logique, constituer trois groupes). 


\section{I.1. Deux pôles de métiers clairement identifiés, qui renvoient à une série d'oppositions convergentes : manuel / non manuel ; privé / public ; production / tertiaire ; etc.}

En première analyse, la constitution des paires laisse entrevoir une représentation fortement clivée de ces métiers, bien éloignée de la situation fictive d'équiprobabilité des vignettes dans chaque groupe (elles seraient alors toutes appariées à $50 \%{ }^{15}$ ). En effet, les professions d'artisan maçon, de soudeur, de réparateur d'ascenseur, de chauffeur livreur, d'ouvrière de découpe de volailles et de caissière sont très souvent associées entre elles dans un même groupe (les taux d'appariement varient de $70 \%$ à $92 \%{ }^{16}$ ) alors qu'elles le sont peu avec les professions de secrétaire de mairie, d'employé de banque et de policier municipal ${ }^{17}$. A l'inverse, ces dernières professions sont fréquemment associées entre elles. Ce clivage est bien illustré par l'ouvrière de découpe de volaille, profession la plus clivante statistiquement de la liste, puisqu'elle est associée plus de neuf fois sur dix avec le chauffeur livreur et le soudeur mais seulement deux fois sur dix avec la secrétaire de mairie. Se dessine ainsi une série de proximités et d'oppositions entre d'une part des métiers à dominante manuelle, essentiellement d'ouvrier, et des métiers d'employé, qui sont rattachés au monde du bureau ou de l'administration.

Une classification ascendante hiérarchique $(\mathrm{CAH})$ réalisée sur les variables indiquant la présence des métiers dans les deux groupes constitués sur la tablette ${ }^{18}$ confirme en les précisant ces premières observations. Une logique de classement principale s'en dégage, que l'on peut qualifier d'hégémonique puisqu'elle concerne $94 \%$ des enquêtés (tableau 1). Cette logique correspond largement aux deux pôles identifiés par les paires de professions : pour les enquêtés qui la suivent, un premier groupe typique regroupe le soudeur, le chauffeur livreur, le réparateur d'ascenseur, l'ouvrière de découpe de volaille et l'artisan-maçon ; le second groupe comprend quant à lui la secrétaire comptable en mairie, le policier municipal, l'agent de service hospitalier et l'employée de banque. L'opposition entre ces deux groupes typiques de la logique hégémonique se retrouve dans le classement le plus fréquemment observé dans l'enquête ( $\mathrm{n}=100$, les métiers d'assistante maternelle et d'agent de service hospitalier étant classés dans le second groupe, qui correspond aux métiers d'employés) et est plus largement présente dans $32 \%$ des classements $(\mathrm{n}=218)^{19}$, si on laisse ouverte la position de l'assistante maternelle et de l'agent de service hospitalier.

Les noms des groupes permettent de comprendre la signification que leur donnent les enquêtés. Le premier groupe typique est le plus souvent désigné par les termes «manuel » $(\mathrm{n}=160)^{20}$, « ouvrier » (103), «pénible » ou «difficile»(32). Les références au secteur "privé » (58), aux activités de «production» ou de «fabrication» (47), ou à «l'artisanat » et aux «artisans » (22) sont également fréquentes. Le caractère dominé de ces métiers transparaît enfin, bien que de façon minoritaire, par exemple lorsque les enquêtés les qualifient d'emplois non ou peu «qualifiés » (25), de métiers «basiques » (18), de « pauvres » ou de « petit salaires » (11), de catégories « modestes » (8), de « classe inférieure » ou «basse»(5). Le second groupe est lui fréquemment intitulé en référence aux

\footnotetext{
${ }^{15}$ Légèrement moins si on permet que des cartes puissent être laissées à part.

${ }^{16}$ A l'exception des artisan-maçon et caissière, qui ne sont appariés que par $59 \%$ des enquêtés. Les pourcentages indiqués dans la suite du texte correspondent à des données pondérées, les effectifs étant parfois indiqués, de façon complémentaire ou alternative (quand le nombre d'observations est faible).

${ }^{17}$ Les taux d'appariement ne dépassent jamais $50 \%$ et se situent majoritairement entre $30 \%$ et $40 \%$.

${ }^{18}$ La classification porte précisément sur les dix premiers axes résultant d'une analyse des correspondances multiples estimée sur les 22 ( 2 x 11) indicatrices de présence de chaque métier dans le premier et le second groupe (les métiers non classés ont des valeurs nulles sur les deux indicatrices). Pour obtenir les logiques de classement finales, on regroupe les classes d'enquêtés dont les premier et second groupes sont symétriques (le choix, arbitraire, de positionner un ensemble de métiers à gauche, respectivement à droite, de l'écran est ainsi neutralisé lors de cette opération).

${ }^{19}$ Contre moins de $0,5 \%$ en probabilité.

${ }^{20}$ Les intitulés ont fait l'objet d'un codage manuel, qui a été permis par la concentration élevée des termes utilisés par les enquêtés. Une cinquantaine de rubriques de registre a été créée (comme « manuel », «basique », etc. pour les métiers d'ouvrier ou employé ; " décideur », "riche, aisé » pour les métiers de cadre ou chef d'entreprise), un même intitulé pouvant renvoyer au plus à trois de ces rubriques. L'utilisation des guillemets rend visible(s) le ou les termes qui leur sont spécifiques. A des fins d'analyse, les rubriques ont parfois été regroupées dans la suite de l'article.
} 
«fonctionnaires » ou au « service public » $(\mathrm{n}=147)$, aux activités de service et plus largement du secteur «tertiaire » (113), à «l'administratif » et au travail de «bureau» (40) ou à la dimension « relationnelle» et d' «aide à la personne» (22). De façon générique, les métiers renvoient aux « employés » (63) et, dans certains cas, à une position supérieure aux métiers du premier groupe : les intitulés sont alors ceux d' « employés qualifiés » (31), de « diplômés » (19), de métiers « intellectuels » ou nécessitant un «savoir» (14), également de métiers peu ou non «pénibles » (14), «stables » ou « protégés » (9), ce qui peut contribuer à en faire des positions, catégories ou classes « moyennes » (22).

Tableau 1 : La principale logique de classement des métiers d'employé et d'ouvrier

\begin{tabular}{|c|c|}
\hline \multicolumn{2}{|l|}{ Premier groupe } \\
\hline Cartes composant le groupe & $\begin{array}{l}\text { Soudeur }(68 \%) \text {, chauffeur livreur }(66 \%) \text {, réparateur d'ascenseur }(65 \%) \text {, } \\
\text { ouvrière de découpe de volaille }(64 \%) \text {, artisan macon }(64 \%)\end{array}$ \\
\hline Carte représentative & $\begin{array}{l}\text { Artisan-maçon }(30 \%) \text {, caissière }(16 \%) \text {, ouvrière de découpe de volaille } \\
(12 \%)\end{array}$ \\
\hline Registres principaux d'intitulé* & $\begin{array}{l}\text { Manuel (160); ouvrier (103); secteur privé (58); activité de production, } \\
\text { fabrication, etc. (47); employé (40); pénible, difficile (32); emploi non ou peu } \\
\text { qualifié }(25) \text {; artisan, artisanal }(22) \text {; divers métier précis }(20) \text {; métier basique } \\
(18) \text {; peu ou non diplômé }(16)\end{array}$ \\
\hline \multicolumn{2}{|l|}{ Second groupe } \\
\hline Cartes utilisées & $\begin{array}{l}\text { Secrétaire comptable }(67 \%) \text {, policier municipal }(62 \%) \text {, agent de service } \\
\text { hospitalier }(61 \%) \text {, employée de banque }(60 \%)\end{array}$ \\
\hline Carte représentative & $\begin{array}{l}\text { Secrétaire comptable mairie }(26 \%) \text {, employée de banque }(21 \%) \text {, policier } \\
\text { municipal }(18 \%) \text {, agent de service hospitalier }(15 \%)\end{array}$ \\
\hline Registres principaux d'intitulé* & $\begin{array}{l}\text { Fonctionnaire, public (147) ; activité de service, tertiaire, etc. (113); employé } \\
\text { (63) ; administratif, bureau (40); emploi qualifié (31) ; relationnel, aide à la } \\
\text { personne (22) ; catégorie moyenne (22) ; diplômé (19); divers métier précis } \\
(17) \text {; jugement négatif (16, planqué, bureaucrate, fainéant, improductif) }\end{array}$ \\
\hline
\end{tabular}

Domaine d'activité (42\%), secteur public-privé (27\%), qualification ( $24 \%$ ), pénibilité $(19 \%)$, statut salarié-indépendant $(15 \%)$

* : ne sont indiqués que les registres d'intitulés correspondant à au moins 15 occurrences. Ils sont présentés par ordre décroissant de fréquence. Les non réponses et réponses imprécises ne sont pas mentionnées.

Champ : ensemble des enquêtés (personnes de plus de 18 ans, France métropolitaine hors Corse) ayant suivi la logique de classement hégémonique ( $94 \%$ de l'ensemble des enquêtés).

Source : enquête «Catégoriser le social » (Elipss, juillet - septembre 2014, n= 733 sur ce champ).

Le clivage que dessinent les intitulés donnés aux deux groupes n'est certes ni tout à fait univoque ni pleinement symétrique ${ }^{21}$. Mais la diversité des noms retenus apparaît largement convergente. Les deux pôles identifiés par la majorité des enquêtés sont de fait désignés par une série d'oppositions qui se recouvrent : manuels / non manuels; ouvriers / employés ; production, fabrication / services, bureau, travail administratif ; privé/public ; pénible / peu pénible ; peu qualifiés / qualifiés ; basique, modeste, inférieur / moyen. Le lexique utilisé converge avec les critères que les enquêtés ont déclaré utiliser pour classer les métiers : le domaine d'activité est le plus fréquemment cité (42\%) devant le secteur (publicprivé, $27 \%$ ), la qualification (24\%) et la pénibilité (19\%). Les responsabilités et le fait d'être chef (ou exécutant) - critères dont on verra qu'ils sont fréquemment évoqués pour les professions de cadres et chefs d'entreprise - sont en revanche peu cités.

Les métiers choisis pour représenter chaque groupe donnent à voir les points saillants de chacun des espaces ainsi délimités. Plus souvent retenues que le policier municipal et l'agent de service, la secrétaire comptable et l'employée de banque montrent le poids symbolique des professions administratives, féminines et qualifiées dans la définition du second groupe. Pour le premier groupe, les métiers fréquemment cités sont plus surprenants : il s'agit de l'artisan-maçon, nettement devant la caissière et l'ouvrière de découpe de volaille; les professions ouvrières masculines (soudeur, réparateur

\footnotetext{
${ }^{21}$ Bien qu'en des proportions peu comparables avec l'usage le plus répandu, les termes « ouvrier » et « employé » sont parfois utilisés à front renversé. Et on peut remarquer que les intitulés du premier groupe donnent une visibilité particulière à la dimension manuelle des métiers concernés, quand ceux du second mettent davantage en avant leur caractère public.
} 
d'ascenseurs, chauffeur-livreur) sont moins souvent choisies. On retrouve ici un résultat mis en avant par L. Boltanski et L. Thévenot (2015 [1983]), qui indiquent que les figures perçues comme typiques des différents groupes professionnels le doivent davantage au travail de représentation dont elles sont l'objet qu'à leur centralité statistique. Dans un ensemble de professions ouvrières salariées visiblement sans un tel point saillant (à l'image du rôle joué par le « Fraiseur P2 » dans le jeu originel), les enquêtés se sont appuyés sur l'artisan-maçon, seul métier exercé en tant qu'indépendant, et sur une profession féminine non ouvrière de caissière. Par la dureté de ses conditions de travail et la faiblesse de ces revenus, l'« ouvrière de découpe de volaille » se situe quant à elle tout en bas de l'échelle des professions, et fait ainsi écho à l'« ouvrière d'usine emballeuse » représentant les travailleurs non qualifiés dans le jeu originel. Ainsi, bien que le terme « ouvrier» reste fortement utilisé pour désigner ce groupe, on ne peut que constater l'absence de professions incarnant de façon consensuelle et saillante le monde ouvrier, et même plus largement l'ensemble des travailleurs « manuels ».

\section{I.2. Métiers frontières ou à la marge : quand certaines situations résistent à la dualité entre « ouvrier » et «employé »}

La perception partagée par une très large majorité d'enquêtés de ces deux pôles distincts de métiers n'épuise pas totalement les logiques de classement adoptées dans le jeu. C'est ce qu'indiquent des logiques minoritaires de classement suivies par $6 \%(\mathrm{n}=49)$ des enquêtés, qui soit n'ont pas voulu ou su jouer le jeu $(n=24)$ - ils ou elles ont classé toutes les cartes dans un unique groupe -, soit ont isolé une carte unique. Cela a concerné pour l'essentiel l'artisan-maçon, et dans une moindre mesure l'employée de banque. Bien que marginales, ces logiques témoignent du statut particulier accordé par une partie des enquêtés à deux métiers considérés comme à la marge de l'espace considéré : l'artisanmaçon compte tenu de son statut d'indépendant, ce qui rappelle l'importance de cette dimension dans la structuration des représentations ordinaires (Hugrée, de Verdalle, 2015) ; l'employée de banque, en raison de son niveau supérieur de revenu et de diplôme, caractéristiques qui rapprochent la profession et plus largement les employées administratives d'entreprise des classes moyennes, en France (Amossé, 2019, à paraître) comme en Europe (Hugrée, Pénissat, Spire, 2017).

D'autres métiers occupent une position particulière dans ce premier jeu. La polarité entre employés et ouvriers comporte en effet des «zones floues », que le raisonnement par paires de professions permet de repérer : certaines professions, que l'on peut qualifier de « frontière » ou «pivot », sont classées de façon variable, étant associées dans des proportions similaires à toutes les autres professions. C'est le cas de l'assistante maternelle et dans une moindre mesure de l'agent hospitalier, vignettes qui sont d'ailleurs souvent associées entre elles (sept fois sur dix). On peut également y ajouter la caissière, qui a par ailleurs souvent été laissée à part $(\mathrm{n}=30)$, bien que moins fréquemment que l'assistante maternelle $(n=43)$. En fonction des caractéristiques auxquelles les enquêtés accordent de l'importance - la dimension relationnelle ou commerciale de l'activité, la dureté des conditions de travail, etc. -, elles peuvent être classées dans un groupe ou dans un autre. Ces professions, plus souvent féminines, appartiennent au monde du tertiaire mais pas des bureaux, s'exercent en intérieur mais leurs conditions de travail - par exemple le fait de travailler debout ou le port de charges lourdes - sont considérées comme pénibles physiquement. Bien que leur nombre ait beaucoup augmenté ces vingt dernières années, leur qualification demeure moins reconnue et identifiée en l'absence d'un travail de représentation politique suffisant : les assistantes maternelles par exemple, peu syndiquées et rarement mobilisées, symbolisent l'invisibilité des emplois à domicile, sans dimension collective forte. Ces emplois manuels et subalternes de service sont à la frontière entre le monde des ouvriers et celui des employés, tel qu'ils se sont historiquement construits. Sans doute constituent-ils un «autre monde populaire », pour reprendre l'expression de Christelle Avril (2014) concernant les aides à domicile, et ainsi un embryon de troisième groupe de métiers au sein de la fraction d'espace social considéré : leur position pivotale et leur classement plus fréquemment à part le suggèrent. Derrière la dualité hégémonique observée, une représentation ternaire leur laissant une place semble pouvoir être envisagée. Le dispositif binaire proposé ne permet toutefois pas aux enquêtés de le mettre pleinement en évidence. 
Finalement, les trois métiers frontières d'assistante maternelle, de caissière et d'agent de service hospitalier et à la marge d'artisan-maçon montrent en creux la force des représentations ordinaires associées au clivage principal opposant les groupes «ouvrier » et «employé » autour des noyaux de métiers que sont d'une part le soudeur, le chauffeur livreur, le réparateur d'ascenseur, l'ouvrière de découpe de volaille et d'autre part la secrétaire administrative de mairie, l'employée de banque et le policier municipal. Le travail manuel, d'extérieur ou pénible, s'oppose encore aujourd'hui clairement aux activités de bureau, ou qui comportent une dimension administrative (comme le policier municipal). Même si aucune profession emblématique ne semble tout à fait s'imposer pour représenter le groupe ouvrier, même si leurs frontières ne font pas pleinement consensus eu égard aux professions peu qualifiés des services, ces deux pôles constituent visiblement le clivage à partir duquel les individus composent leur représentation de ce pan du monde social. Il est loin d'avoir été remplacé par l'opposition entre travailleurs qualifiés et non qualifiés.

\section{I.3. Hiérarchie versus statut : un clivage prenant des significations différentes selon la position sociale des enquêtés}

La logique de classement principalement observée se décline-t-elle exactement de la même manière selon que les enquêtés sont eux-mêmes situés en bas (les ouvriers et les employés) ou en haut (les cadres et professions intermédiaires ${ }^{22}$ ) de l'échelle des emplois salariés ? S'agissant des contours du premier groupe, celui des « ouvriers », les différences sont minimes entre enquêtés. Et il en va de même pour les métiers censés le mieux le représenter. Concernant le second groupe en revanche, si les professions représentatives sont elles aussi similaires, on repère une légère différence concernant les professions le composant : quand les ouvriers et employés tendent à constituer un groupe autour des professions d'employés du public (secrétaire comptable de mairie, agent hospitalier, policier municipal), les cadres et les professions intermédiaires mettent davantage l'accent sur les emplois administratifs plus qualifiés (secrétaire comptable de mairie, employée de banque).

Cette caractérisation des groupes de professions d' "employé » sur la base d'une dimension plus hiérarchique pour les enquêtés cadres et professions intermédiaires trouvent un prolongement dans les noms donnés aux groupes (tableau 2). Concernant le premier groupe, une partie du lexique est utilisée de façon similaire par les enquêtés : «manuel » $(\mathrm{n}=62$ pour les enquêtés ouvriers et employés ; $\mathrm{n}=76$ pour les cadres et professions intermédiaires); secteur «privé » (24 et 26), activité de « fabrication » et de « production » (23 et 20$)$, « pénible » ou « difficile » (16 et 13), métiers « basiques » $(\mathrm{n}=7$ pour les deux types d'enquêtés). Toutefois, les cadres et professions intermédiaires mobilisent plus souvent des termes renvoyant à une hiérarchisation sociale et/ ou professionnelle des positions (emploi non ou peu « qualifié », peu ou non « diplômé », « basique », « pauvre », « exécutant »; cf. tableau 2) alors que les ouvriers et employés insistent davantage sur le secteur public ou privé de l'emploi, l'activité réalisée ou le métier exercé, comme si, par ce biais, ils refusaient de hiérarchiser les distinctions des professions desquelles ils sont les plus proches. Si l'on agrège les termes utilisés pour nommer les groupes, les cadres et professions intermédiaires sont plus nombreux en proportion à utiliser le lexique des PCS ( $24 \%$ versus $19 \%$ pour les ouvriers et employés) et de la hiérarchisation des professions (17\% versus $9 \%$ ) pour le premier groupe. Et ces différences se retrouvent s'agissant du second groupe : les ouvriers et employés insistent plus souvent sur le statut (25\%), et en premier lieu sur le clivage public-privé, que les cadres $(21 \%)$ alors que ces derniers mettent l'accent plus volontiers sur les catégories de la PCS (27\% versus $9 \%)$ et la verticalité des positions (13\% versus $9 \%)$.

\footnotetext{
${ }^{22}$ Le choix de n'inclure les travailleurs indépendants (agriculteurs, artisans, commerçants et chefs d'entreprise) dans aucun de ces deux groupes d'enquêtés tient à leur spécificité et à la forte dispersion de leurs positions sociales, au regard notamment de leurs niveaux de vie et de patrimoine (Bessière, Gollac, 2014).
} 
Tableau 2 : Intitulés et critères utilisés pour classer les métiers d'employé et d'ouvrier selon la position sociale des enquêtés

\begin{tabular}{|c|c|c|}
\hline & $\begin{array}{l}\text { Enquêtés ouvriers et employés } \\
\qquad(\mathrm{n}=\mathbf{3 0 5})\end{array}$ & $\begin{array}{l}\text { Enquêtés cadres et professions } \\
\text { intermédiaires }(n=337)\end{array}$ \\
\hline \multirow{2}{*}{$\begin{array}{l}\text { Intitulés à tonalité « hiérarchique » } \\
\text { utilisés pour désigner le premier } \\
\text { groupe* }\end{array}$} & $\mathrm{n}=32(9 \%)$ & $n=60(17 \%)$ \\
\hline & $\begin{array}{l}\text { Métier basique (7) ; emploi non ou } \\
\text { peu qualifié (5) }\end{array}$ & $\begin{array}{l}\text { Emploi non ou peu qualifié (16); peu } \\
\text { ou non diplômé }(9) \text {; emploi qualifié } \\
(9) \text {; métier basique }(7) \text {; pauvre, petit } \\
\text { salaire }(5) \text {; exécutant }(5)\end{array}$ \\
\hline \multirow{2}{*}{$\begin{array}{l}\text { Intitulés à tonalité « hiérarchique » } \\
\text { utilisés pour désigner le second } \\
\text { groupe* }\end{array}$} & $11=250$ & \\
\hline & $\begin{array}{l}\text { Catégorie moyenne (8); } \\
\text { qualifié (7); intellectuel, s }\end{array}$ & $\begin{array}{c}\text { Emploi qualifié (19) ; diplômé (11); } \\
\text { catégorie moyenne }(10) \text {; intellectuel, } \\
\text { savoir }(7)\end{array}$ \\
\hline Critè & $\begin{array}{c}\text { Domaine d'activité (43\%), secteur } \\
\text { public-privé }(26 \%) \text {, pénibilité }(21 \%) \text {, } \\
\text { qualification }(18 \%) \text {, statut salarié- } \\
\text { indépendant }(18 \%)\end{array}$ & $\begin{array}{l}\text { Domaine d'activité (37 \%), } \\
\text { qualification (35\%), secteur public- } \\
\text { privé }(28 \%) \text {, responsabilité (18\%), }\end{array}$ \\
\hline \multicolumn{3}{|c|}{ : ne sont indiqués que les registres correspondant à au moins 5 intitulés. } \\
\hline \multicolumn{3}{|c|}{$\begin{array}{l}\text { Champ : ensemble des enquêtés (personnes de plus de } 18 \text { ans, France métropolitaine hors Corse) des groupes socio } \\
\text { orofessionnels cadres, professions intermédiaires, employés et ouvriers ayant suivi la logique de classement hégémoniqu } \\
94 \% \text { de l'ensemble des enquêtés). } \\
\text { Source : enquête Catégoriser le social (Elipss, septembre } 2014, \mathrm{n}=642 \text { sur ce champ). }\end{array}$} \\
\hline
\end{tabular}

Ces registres de dénominations sensiblement différents correspondent à des différences de signification attribuée aux groupes réalisés, dont témoignent également les critères utilisés pour classer : plus d'un tiers des cadres et professions intermédiaires (35\%) indiquent avoir privilégié la qualification pour regrouper les métiers, contre moins d'un ouvrier ou employé sur cinq (18\%). Ils sont également plus nombreux à évoquer les responsabilités, là où les ouvriers et employés citent plus volontiers le secteur public-privé, la pénibilité et le statut indépendant-salarié. Le consensus qui se dégage autour de la définition des groupes et clivages internes au monde des ouvriers et des employés n'a donc pas tout à fait la même signification selon la position sociale occupée : si les ouvriers et employés distinguent les professions au regard du contenu des métiers et de leur statut, pour les cadres et professions intermédiaires le clivage s'opère plus volontiers entre les univers professionnels et la qualification des emplois qui leur est associée : redoublant une série d'oppositions dont on trouve la trace dans les intitulés («proche » / « lointain»; «propre » / « sale »; « intérieur » / « extérieur »), ils différencient l'univers du bureau de celui du chantier, de l'usine ou de la route. Les cadres et professions intermédiaires ont ainsi tendance à isoler les employés qualifiés, qu'ils sont susceptibles de côtoyer dans les services administratifs et perçoivent davantage comme des «petits moyens » (Cartier et al., 2008), des ouvriers et employés du service ou du commerce, considérés comme moins qualifiés. Cette catégorisation plus hiérarchisée des métiers qu'adoptent les cadres et professions intermédiaires enquêtés apparaît encore plus nettement dans le second jeu.

\section{Position sociale, statut, fonction : trois manières de regrouper et représenter les métiers de cadre et chef d'entreprise}

A la différence des métiers d'ouvriers et d'employés, ceux de cadres et de chefs d'entreprise n'obéissent pas à une logique dominante de classement. Les groupes réalisés montrent que les différents clivages traversant ces professions se superposent bien moins que dans le premier jeu. L'existence de clivages concurrents tend à brouiller la lisibilité des groupes réalisés, qui sont plus divers. L'analyse de classification permet toutefois de mettre en évidence trois logiques de classement (selon la position sociale, le statut occupé et la fonction exercée), qui sont inégalement adoptées selon la position sociale des enquêtés. 


\section{II.1. Une pluralité des logiques de classement et de dénomination...}

La diversité des regroupements effectués pour les professions de cadre et chef d'entreprise ressort clairement de l'analyse de leurs associations par paire : très peu d'entre elles sont fréquemment associées - seulement cinq paires sont proposées par plus de $70 \%$ des enquêtés, sur 50 possibles -, ou à l'inverse faiblement associées - deux paires le sont par moins de $30 \%$ des enquêtés ${ }^{23}$. Ainsi, les logiques de constitution des groupes sont à première vue plus complexes que dans la séquence précédente. Est-ce à dire que les représentations attachées à ces professions sont plus brouillées et qu'il est plus difficile de se repérer dans l'espace qu'elles délimitent ? Certes, on observe davantage de professions laissées à part que dans la première séquence, comme le préfet $(n=64)$ ou le consultant indépendant $(n=51)$, ce qui peut être interprété comme un indice d'une plus grande difficulté à les classer. Toutefois, les professions non classées peuvent, à l'image du préfet, être des professions par ailleurs bien identifiées et souvent utilisées comme "représentatives » d'un des groupes réalisés : on peut donc plutôt faire l'hypothèse qu'elles sont laissées de côté pour signaler la nécessité de les isoler, parce qu'elles sont considérées comme faisant partie d'un monde à part.

De fait, si elles sont moins nombreuses, les paires de professions fréquemment associées dans un même groupe tendent à confirmer l'existence de plusieurs clivages et pôles identifiables. Trois types d'association se dégagent des paires les plus souvent constituées : le patron (de fabrique de conserve) et le gérant (de magasin de bricolage) définissent un pôle d'indépendants et de chefs d'entreprise ; la directrice des ressources humaines, l'ingénieur informaticien, l'analyste financier dans une grande banque correspondent à un pôle de professionnels au sens anglo-saxon ; le responsable transport dans une mairie, l'inspectrice des impôts et le professeur de français en lycée définissent enfin un pôle de fonctionnaires. Toutefois, ces trois pôles de professions ne suffisent à définir les logiques de classement qui, suivant la consigne du jeu, supposent une partition en deux (et non trois) groupes. De plus, ces trois pôles ne permettent pas de classer les professions restantes (le médecin généraliste, le préfet, le consultant indépendant), qui ont des situations ambivalentes à leur égard : le médecin et le consultant indépendant peuvent être positionnés à la fois avec les professionnels et avec les indépendants ; le préfet n'est visiblement pas uniquement, ni même principalement, perçu comme un fonctionnaire.

Si les pôles qu'esquisse l'analyse des paires de profession ne suffisent à identifier les logiques suivies pour réaliser les classements, une analyse de classification similaire à celle effectuée pour les professions d'ouvriers et d'employés le permet : trois logiques s'en dégagent, dont le poids est réparti de façon relativement équilibrée parmi les enquêtés (tableau 3). Les professions typiques de chacun des groupes associés à ces logiques ont des taux de présence particulièrement élevés, le plus souvent au-delà de $80 \%$, signe de la force des clivages auxquels elles renvoient.

La première logique (37\% des enquêtés) classe les professions selon la position sociale que les enquêtés leur attribuent. Cette représentation positionnelle distingue les cadres moyens des cadres supérieurs : le premier groupe typique regroupe le professeur de français en lycée, le responsable des transports d'une mairie et, dans une moindre mesure, le gérant de magasin de bricolage, quand le second groupe associe fréquemment le préfet et l'analyste financier d'une grande banque. Pour incarner le premier groupe, le professeur de français est le plus cité alors que ce sont le préfet et, dans une moindre mesure, le patron de la fabrique de conserves qui le sont pour le second groupe ${ }^{24}$. De façon cohérente, ces enquêtés déclarent plus souvent avoir utilisé comme critère la responsabilité (41\%), la qualification (33\%), le niveau de revenu (32\%) et le statut d'indépendant ou salarié (26\%), autrement dit plutôt des dimensions

\footnotetext{
${ }^{23} \mathrm{~A}$ titre de comparaison, dans la première séquence on dénombrait 19 paires associées à plus $70 \%$ et 7 paires associées à moins de $30 \%$ pour les professions d'ouvriers et d'employés.

${ }^{24}$ Comme la caissière dans le premier jeu, le fait que le patron de fabrique soit désigné représentatif alors qu'il ne fait pas partie du noyau des professions qui y sont le plus souvent présentes peut être interprété comme une manière pour les enquêtés d'indiquer l'importance particulière de cette profession. Ici, certains joueurs ont pu vouloir signaler que pour eux les positions sociales les plus élevées étaient incarnées par la figure du "patron », même si pour beaucoup d'autres joueurs cette profession a été classée dans d'autres groupes (et selon d'autres logiques, statutaire et fonctionnelle, nous le verrons).
} 
associées à une position élevée dans la hiérarchie sociale. Cette logique fait sens avec le lexique employé pour désigner les groupes, qu'il s'agisse du premier (catégorie ou classe «moyenne », $\mathrm{n}=50$; « intermédiaire », $\mathrm{n}=8$ ) ou du second ( « aisé », « riche », $\mathrm{n}=28$; « dirigeant », « décideur », $\mathrm{n}=23$; « haut placé », « haut responsable », $\mathrm{n}=15$; « classe supérieure », $\mathrm{n}=15$; «(hautement) diplômé », $\mathrm{n}=10$ ). Globalement, $32 \%$ et $44 \%$ des enquêtés ont retenu dans leurs intitulés un terme qui renvoie à une représentation hiérarchisée ${ }^{25}$ des métiers selon leur position dans l'espace social.

Une seconde logique se structure autour d'une opposition statutaire distinguant les professions du public et du privé (32\%) : le premier groupe typique est composé de l'inspecteur des impôts, du préfet, du responsable des transports en mairie et du professeur de français en lycée alors que le second regroupe le patron d'une fabrique de conserves, le gérant magasin bricolage, le consultant indépendant et l'ingénieur informaticien ${ }^{26}$. Le préfet est le métier qui est le plus souvent considéré comme représentatif du premier groupe, tandis que pour le second les réponses sont plus diverses : elles se distribuent entre le médecin généraliste, le patron de fabrique de conserves, le gérant de magasin de bricolage et l'ingénieur informaticien. Le clivage public - privé n'était pas totalement absent de la première logique, comme le montre le statut de métier représentatif qui y était plus souvent accordé au patron de fabrique de conserves par opposition au professeur de français. Mais cette opposition se conjuguait alors à une représentation hiérarchisée socialement, en termes de niveau de vie notamment, de la différence entre les emplois du public et du privé. Le clivage lié à la nature de l'employeur est ici systématisé par les joueurs, comme l'indiquent leurs réponses quant au critère retenu pour leurs classements : une majorité déclare avoir principalement utilisé le secteur public-privé (53\%). Viennent ensuite le domaine d'activité $(30 \%)$ et le statut salarié - indépendant (29\%). Autant que les critères déclarés, ce sont les dénominations les plus communes qui permettent de comprendre le sens donné à cette logique : les termes de «fonctionnaire » ou de « secteur public » $(\mathrm{n}=140)$ désignent massivement le premier groupe alors que ceux de « secteur privé » $(\mathrm{n}=81)$ ou d' « indépendant » $(\mathrm{n}=60)$ sont utilisés pour le second. Ce sont plus globalement $61 \%$ et $58 \%$ des enquêtés ayant suivi cette logique qui ont utilisé un terme renvoyant au statut occupé ${ }^{27}$ pour nommer les premier et second groupes. La forte convergence entre les critères déclarés et la terminologie employée pour désigner les groupes fait d'une certaine manière de cette logique de classement la plus « pure », la plus nette et surtout la plus critérielle des trois.

Une dernière logique met en lumière une représentation fonctionnelle (31 \%) de l'espace des professions considérées, principalement autour de l'opposition entre les encadrants et les experts, ou professionnels, qui est au principe d'une vision anglo-saxonne de la structure des emplois et a été retenue dans la nomenclature européenne EseG (Insee, 2016). Le premier groupe typique se structure autour du gérant de magasin de bricolage, du patron de fabrique de conserves et du responsable des transports de mairie tandis que le second se compose de l'ingénieur informaticien, de l'analyste financier et du médecin généraliste. Le gérant et le patron sont plus souvent choisis pour représenter le premier groupe, ce qui montre la convergence des logiques fonctionnelle et statutaire pour ces professions : les employeurs peuvent être vus à la fois comme des encadrants et comme des indépendants. Pour les enquêtés, le second groupe est quant à lui plus souvent représenté par le médecin, l'analyste financier, mais également le préfet. Cette profession est ainsi considérée comme la plus représentative d'un des groupes dans les trois logiques, signe de sa force symbolique et de son ambivalence : au sein de l'espace considéré, elle est vue à la fois comme emblématique des positions sociales les plus élevées, des emplois de la fonction publique et des experts (en écho à leur excellence scolaire supposée). Les principaux

\footnotetext{
${ }^{25} \mathrm{Il}$ s'agit des rubriques d'intitulés repérant les « décideurs », « dirigeants » ou les « exécutants »; les « riches » ou les « pauvres »; les « haut placés » ou les « modestes », les « petits »; les classes ou catégories « supérieures », «moyennes » ou « basses », « inférieures »; les « intellectuels », « diplômés » ou les « petits diplômes »; les «qualifiés » ou non « qualifiés »; les « cadres » ou les « intermédiaires ».

26162 enquêtés (soit $17 \%$ de l'échantillon pondéré) ont réalisé des groupes opposant ces deux noyaux de professions typiques, alors qu'une situation de pur aléa aurait conduit (en probabilité) à ce choix dans seulement $0,4 \%$ des cas $(1 / \mathrm{C}(4,8)$, avec $\mathrm{C}(4,8)=280)$. Les noyaux correspondant aux représentations positionnelle et fonctionnelle ont respectivement été retenues dans les groupes réalisés par 162 cas ( $21 \%$ en données pondérées, contre $10 \%=1 / \mathrm{C}(2,5)$ en probabilité) et 100 cas $(16 \%$ en données pondérées, contre $2,5 \%=1 / \mathrm{C}(3,6)$ en probabilité).

${ }^{27}$ En l'occurrence les termes ou expressions de «salarié » ou d'« indépendant», «artisan », «profession libérale »; secteur « public » ou « privé ».
} 
critères utilisés selon cette logique sont, sans surprise la qualification (42\%), la responsabilité $(38 \%)$ et le fait d'être chef $(21 \%)$, qui permettent de distinguer les encadrants. Les deux groupes typiques de profession sont désignés par des termes et des expressions plus diversifiées que pour les deux premières logiques, notamment parce qu'elles renvoient à la diversité des métiers ou activités exercées. Le lexique associé au premier groupe mobilise toutefois régulièrement l'expression de «responsabilité » ou de « chef » $(\mathrm{n}=36)$, voire de « dirigeant », « décideur » (14), mais également d' « indépendant » (28). Pour le second, en plus de ceux renvoyant à un univers professionnel spécifique ou à une formation élevée ( « hautement diplômé », $\mathrm{n}=16$ ), les joueurs mobilisent des termes associés à une position élevée (dans l'entreprise comme dans la société : « haut placé », $\mathrm{n}=9$; «classe supérieure », $\mathrm{n}=5$ ) : ce résultat montre le prestige social associé à certaines professions d'expert. Globalement, les termes liés à la fonction exercée ou à la situation professionnelle ${ }^{28}$ sont utilisés par $35 \%$ et $25 \%$ des joueurs pour le premier et second groupe.

${ }^{28}$ Ces termes correspondent aux divers métiers et activités exercés (dont de «bureau », de « service», « relationnel »; « manuelles » ou «non manuelles », « intellectuelles »), aux « décideurs », «dirigeants », « responsables », « chefs », « gérants », «travailleurs, « exécutants », « professions libérales », « cadres », «techniciens », « employés », « ouvriers ». 
Tableau 3 : Logiques de classement et de représentation des professions de chef d'entreprise et de cadre

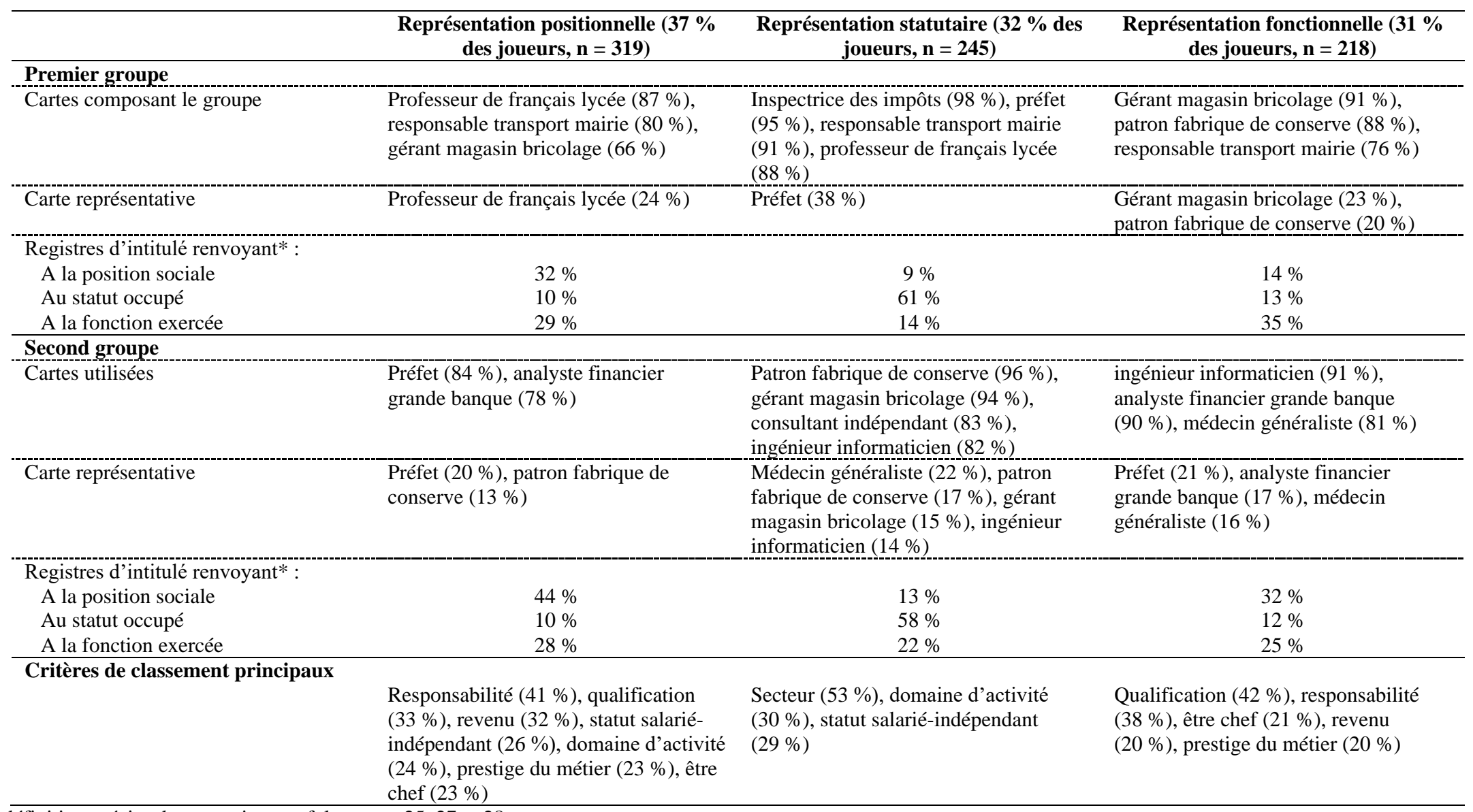

* : pour la définition précise de ces registres, cf. les notes 25,27 et 28 .

Champ : ensemble des enquêtés (personnes de plus de 18 ans, France métropolitaine hors Corse).

Source : enquête Catégoriser le social (Elipss, septembre $2014 ; n=782$ ). 
Les trois logiques de classement qui se dégagent ont une forte cohérence interne : les groupes typiques, dénominations et métiers représentatifs font système. Toutefois, et c'est important de le noter, ces logiques ne sont pas antagoniques; et elles se croisent pour certaines professions de la fraction d'espace social considéré. Le préfet et le patron de fabrique de conserves se révèlent ainsi particulièrement ambivalents : ils sont considérés à la fois du point de vue de leur position sociale (élevée), de leur statut (respectivement public et privé), de leur fonction (respectivement d'expertise et d'encadrement). Il y a bien une pluralité des manières de déclarer et se représenter les professions (Thévenot, 1981 ; Kramarz, 1991), celles proposées dans le jeu en particulier. On peut d'ailleurs remarquer que la pluralité des significations n'empêche pas, au contraire, la force symbolique d'une profession. Comme l'ont montré L. Boltanski et L. Thévenot (2015 [1983]), la représentativité des groupes est le résultat de la visibilité publique ou de la cristallisation de l'image associée à une profession (préfet, médecin) ou à une position (patron).

\section{II.2. ... qui reflètent une représentation inégalement hiérarchisée selon la position et la trajectoire sociales des enquêtés}

Si une logique principale de classement des professions d'ouvrier et d'employé était largement partagée entre enquêtés de positions sociales différentes, il en va bien différemment des professions de cadre et chef d'entreprise. Aussi bien les classements que les dénominations témoignent de représentations différenciées de cette zone de l'espace social.

Comme l'indique le tableau 4, la représentation positionnelle est davantage reprise par les joueurs qui sont eux-mêmes cadres, ou professions intermédiaires, aussi chez ceux qui ont connu une mobilité sociale d'ampleur, et plus particulièrement quand elle est ascendante. Elle est également davantage suivie par les salariés du secteur public et s'accompagne d'un sentiment plus fréquent d'appartenir à une classe sociale. Autrement dit, la représentation hiérarchisée des professions du haut du spectre social est davantage développée par les enquêtés appartenant aux classes moyennes et supérieures, par ceux qui ont gravi les échelons de l'échelle sociale - ils sont sûrement plus sensibles à ce type de perception du fait leur expérience personnelle des différentes positions au sein de ce pan de l'espace social -, et par ceux qui ont une grille de lecture plus politique de la société si l'on se réfère au sentiment qu'ils ont d'appartenir à une classe sociale. La représentation statutaire est davantage adoptée par un tout autre profil de joueurs: des travailleurs indépendants (agriculteurs, artisans, commerçants ou chefs d'entreprise) et des salariés ayant connu une mobilité sociale descendante. Elle s'accompagne d'un intérêt légèrement plus fréquemment déclaré pour la politique, mais sans qu'un sentiment d'appartenir à une classe sociale ne soit plus souvent mentionné. Si la représentation statutaire n'est pas moins politique que celle positionnelle, elle se cristallise chez d'autres joueurs : les indépendants adoptent de façon privilégiée une grille de lecture qui distingue les travailleurs à leur compte des salariés, et plus particulièrement ceux du public ; au-delà d'une différence statutaire, ce clivage correspond pour eux à une distinction voire un antagonisme avec le monde de la bureaucratie et de l'Etat qui structure leurs expériences quotidiennes (Hugrée, de Verdalle, 2015) ${ }^{29}$. Enfin, la représentation fonctionnelle est quant à elle davantage suivie par les employés, et plus encore les ouvriers, ainsi que par les joueurs en situation de reproduction ou d'immobilité sociale, ou encore ceux qui ne déclarent pas avoir le sentiment d'appartenir à une classe sociale. Ainsi, pour organiser les métiers de cadre et chef d'entreprise, les enquêtés qui en sont socialement le plus éloignés se focalisent d'abord sur les fonctions et le contenu des métiers, alors que les enquêtés eux-mêmes cadres, ou professions intermédiaires, les distinguent plus volontiers au regard des hiérarchies professionnelles et sociales.

\footnotetext{
${ }^{29} \mathrm{C}$ 'est autour de cet antagonisme que l'on observe plus particulièrement des intitulés exprimant un jugement sur les métiers à classer, qui restent toutefois largement minoritaires (de l'ordre de quelques pourcents sur les deux séquences) : les « glandeurs », « improductifs », « inutiles », « voleurs de français » sont notamment opposés aux «productifs », «faisants », « entreprenants ». Sans que cela soit systématique, les intitulés les emplois moins qualifiés et/ou du secteur privé sont plus fréquemment associés aux notions de nécessité et de manque de reconnaissance alors que les autres (plus qualifiés, du secteur public) sont désignés avec des connotations qui peuvent être positives ou négatives : ceux qui les occupent ont réussi mais ne le méritent pas toujours.
} 
Tableau 4 : Répartition des trois logiques de classement et de représentation des cartes de cadre et chef d'entreprise selon la position et la trajectoire sociales des enquêtés

\begin{tabular}{|c|c|c|c|}
\hline & $\begin{array}{c}\text { Représentation } \\
\text { positionnelle }(37 \%, n= \\
\text { 319) }\end{array}$ & $\begin{array}{c}\text { Représentation statutaire } \\
(32 \%, \mathrm{n}=\mathbf{2 4 5})\end{array}$ & $\begin{array}{c}\text { Représentation } \\
\text { fonctionnelle }(31 \%, \mathrm{n}= \\
\mathbf{2 1 8}) \\
\end{array}$ \\
\hline \multicolumn{4}{|c|}{$\begin{array}{l}\text { Caractéristiques sociales } \\
\text { où la logique est : }\end{array}$} \\
\hline la plus fréquente & $\begin{array}{l}\text { Cadres }(48 \%) \text {, professions } \\
\text { intermédiaires }(44 \%) ; \\
\text { mobilité sociale ascendante } \\
(59 \%) \text {; a le sentiment } \\
\text { d'appartenir à une classe } \\
\text { sociale }(42 \%)\end{array}$ & $\begin{array}{c}\text { Indépendants }(58 \%) ; \\
\text { mobilité sociale } \\
\text { descendante }(40 \%)\end{array}$ & $\begin{array}{c}\text { Ouvriers }(41 \%) ; \\
\text { trajectoires sociales stables } \\
(37 \%) \text {; n'a pas le } \\
\text { sentiment d'appartenir une } \\
\text { classe sociale }(35 \%)\end{array}$ \\
\hline la moins fréquente & $\begin{array}{c}\text { Ouvrier }(28 \%), \\
\text { indépendants }(32 \%), \\
\text { employés }(35 \%) ; \\
\text { trajectoire sociale stable } \\
(32 \%)\end{array}$ & $\begin{array}{c}\text { Professions intermédiaires } \\
(26 \%) \text {, cadres }(27 \%) ; \\
\text { mobilité sociale ascendante } \\
(21 \%)\end{array}$ & $\begin{array}{c}\text { Indépendants }(10 \%) \text {, cadres } \\
(25 \%) \text {; mobilités sociales } \\
\text { ascendante et descendante } \\
(19 \% \text { et } 21 \%) ;\end{array}$ \\
\hline
\end{tabular}

Champ : ensemble des enquêtés (personnes de plus de 18 ans, France métropolitaine hors Corse).

Source : enquête Catégoriser le social (Elipss, septembre $2014 ; n=782$ ).

Tableau 5 : Intitulés et critères utilisés pour classer les métiers de cadre et chef d'entreprise selon la position sociale des enquêtés

\begin{tabular}{|c|c|c|}
\hline & $\begin{array}{c}\text { Ouvriers et employés } \\
(n=322)\end{array}$ & $\begin{array}{l}\text { Cadres et professions intermédiaires } \\
(n=362)\end{array}$ \\
\hline \multicolumn{3}{|c|}{ Intitulé renvoyant (pour l'un ou l'autre groupe)...* } \\
\hline A la position sociale & $29 \%$ & $44 \%$ \\
\hline Au statut & $36 \%$ & $35 \%$ \\
\hline A la fonction exercée & $42 \%$ & $35 \%$ \\
\hline Critères & $\begin{array}{l}\text { Responsabilité (27\%), statut salarié- } \\
\text { indépendant }(24 \%) \text {, secteur }(23 \%) \text {, } \\
\text { qualification }(22 \%) \text {, domaine } \\
\text { d'activité }(21 \%) \text {, revenu }(19 \%) \text {, } \\
\text { prestige du métier }(17 \%)\end{array}$ & $\begin{array}{l}\text { Qualification }(42 \%) \text {, responsabilité } \\
(37 \%) \text {, secteur }(31 \%) \text {, être chef } \\
(27 \%) \text {, statut salarié-indépendant } \\
(23 \%) \text {, domaine d'activité }(23 \%) \text {, } \\
\text { revenu }(19 \%)\end{array}$ \\
\hline
\end{tabular}

* : pour la définition précise de ces registres, cf. les notes 25,27 et 28.

Champ : ensemble des enquêtés (personnes de plus de 18 ans. France métropolitaine hors Corse) des groupes socioprofessionnels cadres, professions intermédiaires, employés et ouvriers.

Source : enquête Catégoriser le social (Elipss, septembre 2014, n = 684 sur ce champ).

L'analyse des critères utilisés et des dénominations adoptées confirme la nette différence d'importance accordée à la position sociale ou professionnelle par les cadres et professions intermédiaires, et par les ouvriers et employés (tableau 5). Si les intitulés renvoyant à une logique statutaire sont autant évoqués par les uns que les autres, ceux associés à la position sociale le sont bien davantage par les premiers (44\%, contre $29 \%)$, les seconds adoptant préférentiellement des dénominations renvoyant à un registre fonctionnel $(42 \%$, contre $35 \%)$. Les critères retenus par les cadres et professions intermédiaires témoignent également d'une représentation bien plus hiérarchisée de cet ensemble de métiers, notamment sous l'angle de la qualification (ils sont $47 \%$ à citer ce critère, contre $22 \%$ pour les ouvriers et employés), de la responsabilité (37\%, contre $27 \%$ ) et du fait d'être chef $(27 \%$, contre $14 \%)$. Ce poids supérieur accordé aux critères hiérarchisant n'exclut pas d'autres clivages, comme celui du statut (indépendant-salarié), du secteur (public-privé) ou du domaine d'activité, qui sont autant mentionnés (voire davantage pour le secteur, +8 points de pourcentage) par les cadres et intermédiaires. Il y a donc une vision plus hiérarchisée, mais aussi plus complexe, qui se réfère à plus de critères. Le principal critère qui est plus souvent cité par les ouvriers et employés est le prestige du métier (17\%, contre $11 \%$ ), qui montre leur attachement à une dimension symbolique moins objectivante (comme dans l'article de Cédric Hugrée et Laure de Verdalle dans ce numéro). 
La description que fournissent les enquêtés de leur manière de classer les métiers permet de mieux comprendre ces différences. En effet, les cadres et professions intermédiaires déclarent bien plus souvent que les ouvriers et employés utiliser des critères généraux : c'est non seulement le cas lorsqu'ils classent les métiers d'ouvrier et d'employé, dont ils sont socialement éloignés (70 \%, contre $61 \%)$; mais aussi, et même davantage, lorsqu'ils classent les métiers de cadre et chef d'entreprise ( $82 \%$, contre $63 \%)$. A l'inverse, les ouvriers et employés mobilisent plus souvent leur expérience personnelle : pour classer les métiers, $31 \%$ (respectivement $22 \%$ ) indiquent se référer à des personnes qu'ils connaissent ou à leur propre situation dans le premier (respectivement le second) jeu, contre $12 \%$ (respectivement $16 \%$ ) pour les cadres et intermédiaires. Ils citent également plus fréquemment qu'eux l'intuition ou le hasard : $24 \%$ et $21 \%$, contre $16 \%$ et $15 \%$ dans les deux jeux.

Les cadres et professions intermédiaires donnent ainsi l'image de joueurs sérieux, reprenant les critères utilisés pour classer les emplois dans les branches ou les entreprises, tels que la responsabilité ou la qualification. Lorsqu'ils se réfèrent aux catégories socio-professionnelles de la statistique publique, c'est ainsi davantage en référence aux hiérarchies sociales qui les sous-tendent - sur le modèle des grilles à critères classants des conventions collectives -, qu'aux logiques de métiers et à l'intuition des milieux sociaux auxquelles elles renvoient également, qui organisent quant à elles plus fortement les représentations d'ouvriers et d'employés s'appuyant sur leur expérience personnelle, en fonction du type d'activité exercée, des conditions de travail, de l'environnement professionnel et du prestige associé. Ce résultat confirme l'inclinaison inégalement partagée (entre catégories du haut et du bas de l'espace social) à témoigner d'une bonne volonté rationnelle, de type scolaire, plutôt que d'un sens pratique du social adossé à l'expérience ordinaire (Boltanski, Thévenot, 2015 [1983]). Il nous semble plus fondamentalement montrer une divergence sociale dans le regard porté sur la société : cette divergence, qui a traversé l'histoire de la nomenclature socio-professionnelle (Amossé, 2013), renvoie à l'opposition entre classifications «logiques » et «naturelles » (Desrosières, Thévenot, 1979); elle oppose une représentation critérielle et hiérarchisée davantage portée par les cadres et professions intermédiaires à une représentation intuitive, mêlant image sociale et expérience personnelle, qui est plus fréquemment observée chez les employés et les ouvriers. Pour prolonger cette interprétation, et de façon non exclusive, on peut également analyser les réponses des enquêtés comme des manières de prendre position sur le monde social. Tout se passe comme si, avec le jeu, les cadres et professions intermédiaires justifiaient l'ordonnancement des positions internes à la fraction d'espace qu'ils occupent, en lien avec la grandeur de ceux qui l'occupent, celle-ci pouvant être liée aux mérites scolaires, aux niveaux de revenu, aux positions professionnelles élevées ou à l'importance des responsabilités dans la société. Pour les ouvriers et employés, c'est visiblement bien moins souvent le cas.

\section{Conclusion}

Les données de l'enquête mobilisée dans l'article montrent que dans les représentations ordinaires la segmentation entre travailleurs qualifiés et non qualifiés est loin d'avoir remplacé, pour les emplois d'exécution, le clivage distinguant les ouvriers et les employés. Ce résultat interpelle, compte tenu du déclin du monde ouvrier et de la porosité croissante entre activités tertiaires et industrielles, dont témoignent par exemple à la fois le développement des ouvriers de la logistique liés à la grande distribution et l'ouvriérisation des emplois du commerce ou du nettoyage. Il reflète la force toujours actuelle de ces deux groupes symboliques de la nomenclature socio-professionnelle, invitant à la prudence par rapport à l'érosion relevée de son usage (Pierru, Spire, 2008) et à l'annonce de sa moindre pertinence pour décrire les différences sociales (Fitoussi et al., 1996). Plus qu'un métier particulier en leur sein, il semble que ce soient les groupes eux-mêmes, d'ouvrier et d'employé, qui demeurent les principaux points saillants du repérage social dans la fraction basse de l'espace social.

Pour fort et consensuel qu'il soit, ce clivage principal n'est toutefois pas exhaustif, laissant en situation pivotale, à la frontière des deux groupes, certaines professions de service ayant du mal à trouver une place dans l'organisation binaire imposée par le dispositif d'enquête : c'est en particulier le cas des assistantes maternelles, emblématiques des emplois à tonalité domestique, qui peuvent être associées 
aux employés pour leur dimension relationnelle et de service, et aux ouvriers pour leurs conditions de salaire et de travail. La dualité entre ouvrier et employé n'est pas davantage exclusive, agrégeant plusieurs principes de distinction (manuel/non manuel; production/administration, services ; privé / public ; non qualifié / qualifié). L'opposition selon le secteur (entre public et privé) apparaît de façon particulièrement claire à travers la figure des employés du public. Elle joue de façon transversale aux deux jeux, puisqu'adossée aux différences de statut (entre indépendants et salariés) elle correspond à une des trois logiques de classement des métiers de cadre et chef d'entreprise. On mesure en cela le rôle toujours structurant des distinctions sectorielles et statutaires (Hugrée, de Verdalle, 2015), dont on observe la force ici en bas comme en haut de l'espace social.

Pour les métiers de cadre et de chef d'entreprise, les oppositions mises en évidence dans le premier jeu (selon le domaine d'activité, le secteur et le statut, la position) ne se superposent pas, comme c'était le cas entre ouvriers et employés. Trois représentations (positionnelle, statutaire et fonctionnelle) se dégagent ainsi, dont la première est clairement hiérarchisée. Cette manière d'organiser les professions apparaît particulièrement nette dans les critères utilisés pour classer et peut être reliée aux caractéristiques des enquêtés (ce qui est un apport original du dispositif): la responsabilité, la qualification et le revenu apparaissent déterminants pour classer les métiers de cadre et de chef d'entreprise ; surtout, ils sont très inégalement mobilisés selon la position sociale des enquêtés. C'est ainsi à une double asymétrie que concluent les données : les hiérarchies professionnelles et sociales sont plus souvent évoquées pour et par les individus occupant une position élevée alors que ce sont davantage des oppositions transversales (statutaires ou fonctionnelles) qui sont utilisées pour et par ceux situés plus bas dans l'échelle des emplois et des positions sociales.

Ces conclusions nous semblent pouvoir être reliées à l'inégale diffusion des grilles à critères classants et des méthodes managériales d'évaluation des emplois, auxquelles les cadres et professions intermédiaires sont davantage exposés. Elles témoignent en tous cas des différences fortes dans les représentations ordinaires selon la position sociale, les ouvriers et employés restant visiblement plus attachés aux logiques de métiers qu'à une lecture hiérarchisée du monde social.

\section{Bibliographie}

Amossé Thomas, « Cadre-non cadre : une frontière toujours consistante » in Paul Bouffartigue, Michel Forsé, Sophie Pochic (dir.), Cadres-classes moyennes : vers l'éclatement, Armand Colin, 2011.

Amossé Thomas, «La nomenclature socio-professionnelle : une histoire revisitée », Annales, Histoire Sciences Sociales, 68-4, 2013, p. 1039-1075

Amossé Thomas, «Quelles catégorisations socio-professionnelles des classes populaires? (à paraître).

Amossé Thomas et Chardon Olivier, «Les travailleurs non qualifiés : une nouvelle classe sociale ? », Economie et Statistique, n³93-394, 2006, p. 203-229.

Avril Christelle, Les aides à domicile. Un autre monde populaire, La dispute, 2014.

Bessière C., Gollac S. (2014), «Travailleurs indépendants » in Pierre-Michel Chauvin, Michel Grossetti, Pierre-Paul Zalio (dir.), Dictionnaire sociologique de l'entrepreneuriat, Presses de Sciences Po, p. 537-550.

Boltanski Luc et Chiapello Eve, Le nouvel esprit du capitalisme, Gallimard, 1999.

Boltanski Luc et Thévenot Laurent, «Comment s'orienter dans le monde social », Sociologie, vol. 6, $\mathrm{n}^{\circ} 1,2015$, p. 5-30, traduction de « Finding one's Way in Social Space: a study based on games », Social Science Information, vol. 22, n4-5, 1983, p. 631-679.

Bouffartigue Paul, La fin d'une figure sociale, La dispute, 2001, 246 p. 
Brousse Cécile, «Réflexions sur la nomenclature socio-économique européenne en gestation », Revue Française de Socio-économie, n 10 , 2012, p. 241-250.

Burnod Guillaume et Chenu Alain, «Les représentations ordinaires de la division du travail : une étude fondée sur les déclarations de profession », in Pierre-Michel Menger (dir.), Les professions et leurs sociologies, Editions de la MSH, 2003, p. 119-133.

Cartier Marie, Coutant Isabelle, Masclet Olivier, Siblot Yasmine, La France des «petits-moyens». Enquête sur la banlieue pavillonnaire, La Découverte, 2008.

Chambaz Christine, Maurin Eric, Torelli Constance, «L'évaluation sociale des professions en France. Construction et analyse d'une échelle des professions », Revue française de sociologie, 39-1, 1998, p. 177-226.

Chenu A. (1990), L'archipel des employés, Insee, collection Études.

Coutrot Laurence, «Archéologie des logiques de compétences », L'Année sociologique, Vol. 55, $\mathrm{n}^{\circ} 1$, 2005, p. 197-230.

Deauvieau Jérôme, Pénissat Étienne, Brousse Cécile et Jayet Cyril, « Les catégorisations ordinaires de l'espace social français. Une analyse à partir d'un jeu de cartes », Revue française de sociologie, vol. 55, $\mathrm{n}^{\circ} 3$, 2014, p. 411-457.

Desrosières Alain, Thévenot Laurent, «Les mots et les chiffres: les nomenclatures socioprofessionnelles, Economie et statistique, n¹10, 1979, p. 49-65.

Desrosières Alain, Thévenot Laurent, Les catégories socio-professionnelles, La découverte, 2002 [1988], $128 \mathrm{p}$.

Fitoussi Jean-Paul et Rosanvallon Pierre, Le Nouvel Âge des inégalités, Seuil, 1996.

Goldthorpe John, «Social Class and the Differentiation of Employment», in Goldthorpe John, On Sociology, Numbers, Narratives and the Integration of Research and Theory, Oxford University Press, 2000.

Hugrée Cédric, de Verdalle Laure, " "Fonctionnaires" et "indépendants" à l'épreuve des catégorisations ordinaires du monde social », Sociologie du travail, 2015, 57-2, p. 200-229.

Hugrée Cédric, Pénissat Etienne, Spire Alexis, «Les différences entre salariés du public et du privé après le tournant managérial des États en Europe », Revue Française de Sociologie, vol. 56, 2015, p. 47-73.

Hugrée Cédric, Pénissat Etienne, Spire Alexis, Les classes sociales en Europe, Agone, 2017, 264 p.

Insee, « $\mathrm{ESeG}=$ European Socio economic Groups. Nomenclature socio-économique européenne », Document de travail, $\mathrm{n}^{\circ} 1604,2016$.

Kramarz Francis, « Déclarer sa profession », Revue française de sociologie, n³2-1, 1991, pp. 3-27.

Pénissat Étienne, Brousse Cécile et Deauvieau Jérôme, «Finding one's way in social space : genèse, postérité et actualité d'une enquête originale », Sociologie, vol. 6-1, 2015, p. 31-42.

Pénissat Étienne, Brousse Cécile et Deauvieau Jérôme, Chevillard Julien, Barozet Emmanuelle and Mac-Clure Oscar, «From Statistical Categorizations to Ordinary Categorizations of Social Space: History and Legacy of an Original Study Based on a Card Game », Historical Social Research, vol. 41, 2016, p. 135-154.

Pierru Emmanuel, Spire Alexis, « Le crépuscule des catégories socioprofessionnelles », Revue française de science politique, vol. 58, no. 3, 2008, pp. 457-481. 
Tallard Michèle, «L'introduction de la notion de compétence dans les grilles de classification : genèse et évolution », Sociétés Contemporaines, n41-42, 2001, p. 159-187.

Thévenot Laurent, «Un emploi à quel titre ? L'identité professionnelle dans les enquêtes statistiques », Archives et documents, vol. XXXVIII, Les catégories socioprofessionnelles et leur repérage dans les enquêtes. Études méthodologiques, Insee, 1981, pp. 9-39.

Vigna Xavier, Histoire des ouvriers en France au XXème siècle, Perrin, 2012, 468 p. 